\title{
MIXING SEQUENCES IN HILBERT SPACES
}

\author{
DANIEL BEREND AND VITALY BERGELSON
}

\begin{abstract}
A general setup is provided for theorems of the Blum-Hanson type. Notions of strongly and weakly mixing sequences of elements in a Hilbert space are defined, and equivalent characterizations are derived. It turns out that many known generalizations of the Blum-Hanson theorem are special cases of an abstract version which is also suitable for the nonlinear situation.
\end{abstract}

Introduction. In [4] the notions of ergodicity, weak mixing, and strong mixing were defined and studied for sequences of measure preserving transformations of probability spaces. These notions generalize the classical ones (where a transformation $T$ is identified with the sequence $\left.\left(T^{n}\right)_{n=0}^{\infty}\right)$. It turns out that many results, which are well known for sequences of powers of a single transformation remain valid in the sequential setup. For example, a sequence $\left(T_{n}\right)$ is strongly mixing iff

$$
\frac{1}{N} \sum_{k=1}^{N} T_{n_{k}} f \underset{N \rightarrow \infty}{\stackrel{L^{p}}{\rightarrow}} \int_{X} f d \mu, \quad f \in L^{p},
$$

for every increasing sequence $\left(n_{k}\right)$ [4, Theorem 3.1]. This generalizes a characterization of strongly mixing transformations given by Blum and Hanson [5]. Also, $\left(T_{n}\right)$ is weakly mixing iff the aforementioned convergence holds for every sequence $\left(n_{k}\right)$ of positive lower density [4, Theorem 4.1]. This forms a generalization of a result of Jones [7].

In $[1,6,8]$, results of the Blum-Hanson type were obtained for contractions of Hilbert spaces. In view of [4] the question arises whether these results admit generalizations to sequences of contractions. In this paper we settle this question in the affirmative and show that, indeed, much more is true. Namely, one can satisfactorily define the notions of strong and weak mixing for arbitrary sequences in Hilbert spaces; many results concerning mixing properties of measure preserving transformations or contractions of Hilbert spaces are special cases of more general (yet simple enough) properties of mixing sequences of elements in Hilbert spaces.

The above notions can also be studied for more general spaces. However, this is a

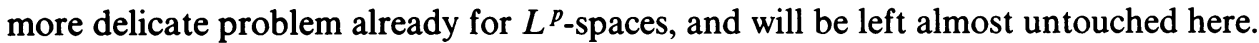
We refer to $[2,3,9]$ for results in more general spaces.

$\$ 1$ deals with strongly mixing sequences. The results of this section are used in $\$ 2$ to obtain simplified proofs of generalized versions of some of the results of $[1,5,10]$. In $\$ 3$ we study weakly mixing sequences.

We would like to thank L. Sucheston for helpful discussions related to this topic.

Received by the editors August 29, 1985.

1980 Mathematics Subject Classification. Primary 47A35; Secondary 28D99, 46C99. 
1. Strongly mixing sequences in Hilbert spaces. Throughout the paper, $\mathscr{H}$ denotes a Hilbert space and $\left(f_{n}\right)_{n=1}^{\infty}$ a sequence in $\mathscr{H}$. We denote by $|P|$ the number of elements of a finite set $P$.

THEOREM 1.1. Let $\left(f_{n}\right)$ be a bounded sequence. Then the following conditions are equivalent:

(1) For any $\varepsilon>0$ there exists a $K=K(\varepsilon)$ such that for any $g \in \mathscr{H}$ with $\|g\| \leqslant 1$ there exists a set $P \subseteq \mathbf{N}$ with $|P| \leqslant K$ such that

$$
\left|\left(f_{n}, g\right)\right| \geqslant \varepsilon \Rightarrow n \in P \text {. }
$$

(2) For any $\varepsilon>0$ there exists a $K=K(\varepsilon)$ such that for any $m \in \mathbf{N}$ there exists $a$ set $P \subseteq \mathbf{N}$ with $|P| \leqslant K$ such that

$$
\left|\left(f_{n}, f_{m}\right)\right| \geqslant \varepsilon \Rightarrow n \in P .
$$

(3) For any $\varepsilon>0$ there exists an $L=L(\varepsilon)$ such that, if $N>L$ and $n_{1}<n_{2}<\cdots$ $<n_{N}$ are positive integers, then

$$
\left\|\frac{1}{N} \sum_{k=1}^{N} f_{n_{k}}\right\|<\varepsilon .
$$

(4) For any increasing sequence $\left(n_{k}\right)$

$$
\left\|\frac{1}{N} \sum_{k=1}^{N} f_{n_{k}}\right\| \underset{n \rightarrow \infty}{\rightarrow} 0 .
$$

Proof. We may assume that $\left\|f_{n}\right\| \leqslant 1$ for each $n$.

(2) $\Rightarrow$ (3) Let $\varepsilon>0$. Take $K=K\left(\varepsilon^{2} / 2\right)$ as in condition (2), $L>2 K / \varepsilon^{2}$. If $N>L$ and $n_{1}<n_{2}<\cdots<n_{N}$, then

$$
\begin{aligned}
\left\|\frac{1}{N} \sum_{k=1}^{N} f_{n_{k}}\right\|^{2} & \leqslant \frac{1}{N^{2}} \sum_{j, k=1}^{N}\left|\left(f_{n_{j}}, f_{n_{k}}\right)\right| \leqslant \frac{1}{N^{2}}\left(K N+\frac{N^{2} \varepsilon^{2}}{2}\right) \\
& <\frac{K}{L}+\frac{\varepsilon^{2}}{2}<\varepsilon^{2}
\end{aligned}
$$

so that $\left\|(1 / N) \sum_{k=1}^{N} f_{n_{k}}\right\|<\varepsilon$.

(4) $\Rightarrow$ (1) Suppose (1) does not hold. We can then find $\varepsilon>0$ such that for each $m \in \mathbf{N}$ there exists a $g_{m} \in \mathscr{H}$ with $\left\|g_{m}\right\| \leqslant 1$ and a set $P_{m} \subseteq \mathbf{N}$ such that $\left|P_{m}\right| \underset{m \rightarrow \infty}{\rightarrow} \infty$ and

$$
\left|\left(f_{n}, g_{m}\right)\right| \geqslant \varepsilon, \quad n \in P_{m} .
$$

Replacing each $P_{m}$ by a subset thereof we may assume that for each $m$ all the numbers $\left(f_{n}, g_{m}\right), n \in P_{m}$, lie in the same quadrant in the complex plane. We have therefore

$$
\left\|\frac{1}{\left|P_{m}\right|} \sum_{n \in P_{m}} f_{n}\right\| \geqslant \frac{1}{\left|P_{m}\right|}\left|\sum_{n \in P_{m}}\left(f_{n}, g_{m}\right)\right| \geqslant \frac{\varepsilon}{4} \text {. }
$$


Notice that it may also be assumed that the sequence $\left(\left|P_{m}\right|\right)_{m=1}^{\infty}$ grows arbitrarily fast and that $\max P_{m}<\min P_{m+1}$ for each $m$. It then follows from (1.1), denoting by $\left(n_{k}\right)$ the increasing sequence formed by the elements of $\cup_{n=1}^{\infty} P_{m}$, that

$$
\left\|\frac{1}{N} \sum_{k=1}^{N} f_{n_{k}}\right\| \underset{N \rightarrow \infty}{\stackrel{\leftrightarrow}{\nrightarrow}} 0,
$$

which contradicts (1).

Since the implications (1) $\Rightarrow(2)$ and $(3) \Rightarrow(4)$ are trivial, this completes the proof.

Definition 1.1. A bounded sequence $\left(f_{n}\right)_{n=1}^{\infty}$ in $\mathscr{H}$ is strongly mixing if it satisfies any of the conditions (1)-(4) in Theorem 1.1.

2. Applications. (i) Let $(X, \mathscr{B}, \mu)$ be a probability space and $T$ a measure preserving transformation thereof. Denote by $U$ the corresponding isometry of $L^{2}(X, \mathscr{B}, \mu)$. Let $f \in L^{2}(X, \mathscr{B}, \mu)$ with $\int_{X} f d \mu=0$ and $\left(k_{n}\right)$ be an increasing sequence of positive integers. Set $f_{n}=U^{k_{n}} f$. It is easy to see that if $T$ is strongly mixing, then $\left(f_{n}\right)$ satisfies condition (2) of Theorem 1.1. Condition (3) of the theorem yields now a strengthened version of the Blum-Hanson theorem [5].

(ii) Suppose that $\left(T_{n}\right)$ is a sequence of measure preserving transformations of a probability space $(X, \mathscr{B}, \mu)$. Denote by $\mathscr{H}_{0}$ the subspace of $L^{2}(X, \mathscr{B}, \mu)$ consisting of those functions $f$ with $\int_{X} f d \mu=0$. One readily verifies that $\left(T_{n}\right)$ is a strongly mixing sequence (in the sense of [4, Definition 3.2]) iff for every $f \in \mathscr{H}_{0}$ the sequence $\left(T_{n} f\right)$ satisfies condition (2) of Theorem 1.1. This theorem shows therefore that our notion of strong mixing forms an abstract generalization of the notion of strong mixing of sequences of measure preserving transformations. As an example let us mention that if $\left(n_{k}\right)$ is any sequence of positive integers, then the sequence $\left(T_{k}\right)$ of transformations of $[0,1)$ into itself given by

$$
T_{k} x=n_{k} x \quad(\bmod 1), \quad x \in[0,1),
$$

is strongly mixing (this follows as a special case from [4, Theorem 5.5]). Hence for every $f \in \mathscr{H}_{0}$ the sequence $\left(T_{k} f\right)$ is strongly mixing, which easily implies that for every $f \in L^{2}[0,1)$ we have

$$
\frac{1}{N} \sum_{k=1}^{N} f\left(\left\{n_{k} x\right\}\right) \underset{N \rightarrow \infty}{\stackrel{L^{2}}{\rightarrow}} \int_{0}^{1} f(t) d t
$$

(this was first proved by Raikov [11]). For generalizations to sequences of epimorphisms of compact groups see $[4, \S 5]$.

(iii) Let $(X, \mathscr{B}, \mu)$ be a $\sigma$-finite infinite measure space. Call a measure preserving transformation $T$ strongly mixing if for every $A \in \mathscr{B}$ with $\mu(A)<\infty$ we have $\mu\left(A \cap T^{-n} A\right) \underset{n \rightarrow \infty}{\rightarrow} 0$. A theorem of Krengel and Sucheston [10, Theorem 4.1] states that, if $1<p \stackrel{n \rightarrow \infty}{<\infty}$, then $T$ is strongly mixing iff for every $f \in L^{p}(X, \mathscr{B}, \mu)$ and $\varepsilon>0$ there exists an $L$ such that if $N>L$ and $n_{1}<n_{2}<\cdots<n_{N}$ are positive integers, then

$$
\left\|\frac{1}{N} \sum_{k=1}^{N} T^{n_{k}} f\right\|<\varepsilon .
$$


We shall now show that the theorem extends to the sequential setup. First we need the following

DEFINITION 2.1. Let $\left(T_{n}\right)$ be a sequence of measure preserving transformations of a $\sigma$-finite infinite measure space $(X, \mathscr{B}, \mu)$. The sequence $\left(T_{n}\right)$ is strongly mixing if for any $A \in \mathscr{B}$ with $\mu(A)<\infty$ and any $\varepsilon>0$ there exists a $K=K(\varepsilon)$ such that for any $m \in \mathbf{N}$ there exists a set $P \subseteq N$ with $|P| \leqslant K$ such that

$$
\mu\left(T_{m}^{-1} A \cap T_{n}^{-1} A\right) \geqslant \varepsilon \Rightarrow n \in P .
$$

We note that in the terminology of [4, Definition 3.1] the last condition amounts to saying that the set $\left\{(m, n): \mu\left(T_{m}^{-1} A \cap T_{n}^{-1} A\right) \geqslant \varepsilon\right\}$ is of bounded fibers.

THEOREM 2.1. Let $\left(T_{n}\right)$ be a sequence of measure preserving transformations of a $\sigma$-finite infinite measure space $(X, \mathscr{B}, \mu)$ and $1<p<\infty$. Then $\left(T_{n}\right)$ is strongly mixing iff for every $f \in L^{p}(X, \mathscr{B}, \mu)$ and $\varepsilon>0$ there exists an $L$ such that, if $N>L$ and $n_{1}<n_{2}<\cdots<n_{N}$ are positive integers, then

$$
\left\|\frac{1}{N} \sum_{k=1}^{N} T_{n_{k}} f\right\|_{p}<\varepsilon .
$$

In fact, we readily see that $\left(T_{n}\right)$ is strongly mixing iff every sequence of the form $\left(T_{n} 1_{A}\right), A \in \mathscr{B}$ with $\mu(A)<\infty$, considered as a sequence in $L^{2}(X, \mathscr{B}, \mu)$, satisfies condition (2) of Theorem 1.1. The equivalence (2) $\Leftrightarrow(3)$ of that theorem yields therefore the case $p=2$ in our theorem. For arbitrary $1<p<\infty$, the "if" part follows from the case $p=2$ employing elementary inequalities, while the proof of the "only if" part follows that of the corresponding part in the Krengel-Sucheston theorem (see [10, pp. 159-160] and will not be elaborated here.

(iv) Our last application is the following version of a result of Akcoglu-Sucheston [1] and Jones-Kuftinec [8].

THEOREM 2.2. Let $T$ be a contraction of a Hilbert space $\mathscr{H}$. Then the following conditions are equivalent:

(1) $T^{n} f \underset{n \rightarrow \infty}{\rightarrow} 0$ weakly for every $f \in \mathscr{H}$.

(2) $(1 / N) \sum_{k=1}^{N} T^{n_{k}} \underset{n \rightarrow \infty}{\rightarrow} 0$ strongly for every $f \in \mathscr{H}$ and increasing sequence $\left(n_{k}\right)$.

Proof. Let $f \in \mathscr{H}$. If $(1 / N) \sum_{k=1}^{N} T^{n_{k}} \underset{N \rightarrow \infty}{\rightarrow} 0$ strongly for every increasing $\left(n_{k}\right)$, then $\left(T^{n} f\right)$ satisfies condition (4) of Theorem 1.1, so that using condition (1) there we see that $T^{n} f \underset{n \rightarrow \infty}{\rightarrow} 0$ weakly. Thus $(2) \Rightarrow(1)$. On the other hand, if $T^{n} f \underset{n \rightarrow \infty}{\rightarrow} 0$ weakly, then one can easily show as in [1] that for any $\varepsilon>0$ there exists a $K$ such that if $|n-m| \geqslant K$ then $\left|\left(T^{m} f, T^{n} f\right)\right| \leqslant \varepsilon$. This shows that the sequence $\left(T^{n} f\right)$ satisfies condition (2) of Theorem 1.1, whence by (4) there $(1 / N) \sum_{k=1}^{N} T^{n_{k}} \underset{n \rightarrow \infty}{\rightarrow} 0$ strongly for every increasing $\left(n_{k}\right)$. Thus $(1) \Rightarrow(2)$. This proves the theorem. 
Notice that we could have avoided employing the inequality from [1] in the last proof by using dilations, and thus reducing the theorem to the case of unitary operators.

3. Weakly mixing sequences. In this section we shall deal mainly with weakly mixing sequences. A few definitions, in particular that of ergodic sequences, are required first.

Proposition 3.1. Let $\left(f_{n}\right)$ be a bounded sequence in a Hilbert space $\mathscr{H}$. Then the following conditions are equivalent:

(1) There exists a sequence $\left(\varepsilon_{N}\right)$ of positive numbers converging to 0 such that

$$
\left|\frac{1}{N} \sum_{n=1}^{N}\left(f_{n}, g\right)\right| \leqslant \varepsilon_{N}\|g\|, \quad g \in \mathscr{H} .
$$

(2) $(1 / N) \sum_{n=1}^{N} f_{n} \underset{N \rightarrow \infty}{\rightarrow} 0$ strongly in $\mathscr{H}$.

The proof is straightforward.

Definition 3.1. A bounded sequence $\left(f_{n}\right)$ in a Hilbert space $\mathscr{H}$ is ergodic if it satisfies either one of the two conditions in Proposition 3.1.

REMARK 3.1. With this terminology, the last condition in Theorem 2.1 can be replaced by:

(4') Every subsequence of $\left(f_{n}\right)$ is ergodic.

An increasing sequence of positive integers $\left(n_{k}\right)$ is of positive lower density if $n_{k}=O(k)$.

Given a Hilbert space $\mathscr{H}$, we denote by $\overline{\mathscr{H}}$ its conjugate, namely the space given by:

(1) As an additive group, $\overline{\mathscr{H}}$ is the same as $\mathscr{H}$.

(2) Multiplication by a scalar is defined by

$$
\lambda \circ f=\bar{\lambda} f, \quad \lambda \in \mathbf{C}, f \in \mathscr{H} .
$$

(3) The inner product is defined by

$$
\langle f, g\rangle=(\overline{f, g}), \quad f, g \in \mathscr{H} .
$$

For Hilbert spaces $\mathscr{H}$ and $\mathscr{K}$, we denote by $\mathscr{H} \otimes \mathscr{K}$ the tensor product of the spaces and by $f \otimes g$ the product of $f \in \mathscr{H}$ and $g \in \mathscr{K}$.

THEOREM 3.1. Let $\left(f_{n}\right)$ be a bounded sequence in $\mathscr{H}$. Then the following conditions are equivalent:

(1) $\left(1 / N^{2}\right) \sum_{m, n=1}^{N}\left|\left(f_{m}, f_{n}\right)\right| \underset{N \rightarrow \infty}{\rightarrow} 0$.

(2) For any $\delta, \varepsilon>0$ there exists an $L$ such that for every $N \geqslant L$ and $m$ the inequality $\left|\left(f_{m} f_{n}\right)\right| \geqslant \varepsilon$ has at most $\delta N$ solutions $n$ with $1 \leqslant n \leqslant N$.

(3) The sequence $\left(f_{n_{k}}\right)$ is ergodic for every positive lower density sequence $\left(n_{k}\right)$.

(4) The sequence $\left(f_{n} \otimes f_{n}\right)$ is ergodic in $\mathscr{H} \otimes \overline{\mathscr{H}}$.

(5) For any bounded sequence $\left(g_{n}\right)$ in a Hilbert space $\mathscr{K}$, the sequence $\left(f_{n} \otimes g_{n}\right)$ is ergodic in $\mathscr{H} \otimes \mathscr{K}$. 
Proof. (1) $\Rightarrow$ (3) Let $\left(n_{k}\right)$ be of positive lower density. For an appropriately chosen positive integer $C$ we have $n_{k} \leqslant C k$ for each $k$. Then

$$
\begin{aligned}
\left\|\frac{1}{N} \sum_{n=1}^{N} f_{n_{k}}\right\|^{2} & =\frac{1}{N^{2}} \sum_{i, j=1}^{N}\left(f_{n_{i}}, f_{n_{j}}\right) \leqslant \frac{1}{N^{2}} \sum_{m, n=1}^{C N}\left|\left(f_{m}, f_{n}\right)\right| \\
& =C^{2} \cdot \frac{1}{(C N)^{2}} \sum_{m, n=1}^{C N}\left|\left(f_{m}, f_{n}\right)\right| \underset{N \rightarrow \infty}{\rightarrow} 0
\end{aligned}
$$

and so $(1 / N) \sum_{k=1}^{N} f_{n_{k}} \underset{N \rightarrow \infty}{\rightarrow} 0$.

(3) $\Rightarrow$ (2) Assume that (2) is not satisfied. Similar to the proof of the implication (4) $\Rightarrow(1)$ in Theorem 1.1, we can construct increasing sequences $\left(n_{k}\right)$ and $\left(N_{j}\right)$ such that $n_{N_{j}}=O\left(N_{j}\right)$ and

$$
\underset{j \rightarrow \infty}{\lim _{j \rightarrow \infty}}\left\|\frac{1}{N_{j}} \sum_{k=1}^{N_{j}} f_{n_{k}}\right\|>0 .
$$

Expand the sequence $\left(n_{k}\right)$ by adjoining to it all the multiples of a certain positive integer $d$. The resulting sequence is of positive lower density, and from (3.1) it follows that, provided $d$ is large enough, the corresponding subsequence of $\left(f_{n}\right)$ is nonergodic.

(1) $\Rightarrow(5)$ Let $\left(g_{n}\right)$ be a bounded sequence in $\mathscr{K}$. Suppose, say, that $\left\|g_{n}\right\| \leqslant 1$ for all $n$. Then

$$
\begin{aligned}
\left\|\frac{1}{N} \sum_{n=1}^{N} f_{n} \otimes g_{n}\right\|^{2} & =\frac{1}{N^{2}} \sum_{m, n=1}^{N}\left(f_{m} \otimes g_{m}, f_{n} \otimes g_{n}\right) \\
& =\frac{1}{N^{2}} \sum_{m, n=1}^{N}\left(f_{m}, f_{n}\right)\left(g_{m}, g_{n}\right) \\
& \leqslant \frac{1}{N^{2}} \sum_{m, n=1}^{N}\left|\left(f_{m}, f_{n}\right)\right| \underset{N \rightarrow \infty}{\rightarrow 0} 0
\end{aligned}
$$

whence $\left(f_{n} \otimes g_{n}\right)$ is ergodic.

(4) $\Rightarrow$ (1) Denote by $(\cdot, \cdot),\langle\cdot, \cdot\rangle$ and $\langle\langle\cdot, \cdot\rangle\rangle$ the scalar product in $\mathscr{H}, \overline{\mathscr{H}}$ and $\mathscr{H} \otimes \overline{\mathscr{H}}$, respectively. Since $\left(f_{n} \otimes f_{n}\right)$ is ergodic in $\mathscr{H} \otimes \overline{\mathscr{H}}$ we have

$$
\begin{aligned}
\frac{1}{N^{2}} \sum_{m, n=1}^{N}\left|\left(f_{m}, f_{n}\right)\right|^{2} & =\frac{1}{N^{2}} \sum_{m, n=1}^{N}\left(f_{m}, f_{n}\right)\left\langle f_{m}, f_{n}\right\rangle \\
& =\frac{1}{N^{2}} \sum_{m, n=1}^{N}\left\langle\left\langle f_{m} \otimes f_{m}, f_{n} \otimes f_{n}\right\rangle\right\rangle \\
& =\left\|\frac{1}{N} \sum_{n=1}^{N} f_{n} \otimes f_{n}\right\|^{2} \underset{N \rightarrow \infty}{\rightarrow} 0 .
\end{aligned}
$$


In view of [4, Lemma 4.1] this implies

$$
\frac{1}{N^{2}} \sum_{m, n=1}^{N}\left|\left(f_{m}, f_{n}\right)\right| \underset{N \rightarrow \infty}{\rightarrow} 0 .
$$

The implications $(2) \Rightarrow(1)$ and $(5) \Rightarrow(4)$ being straightforward, this completes the proof.

Definition 3.2. A bounded sequence $\left(f_{n}\right)$ in a Hilbert space $\mathscr{H}$ is weakly mixing if it satisfies either one of the conditions in Theorem 3.1.

Our results in $\$ 2$ admit analogues to the case of weak mixing. Before giving an illustration, we need the following

DEFINITION 3.3. A sequence $\left(T_{n}\right)$ of measure preserving transformations of a $\sigma$-finite infinite measure space $(X, \mathscr{B}, \mu)$ is ergodic (resp. weakly mixing) if for every $f \in L^{2}(X, \mathscr{B}, \mu)$ the sequence $\left(T_{n} f\right)$ is ergodic (resp. weakly mixing).

It is routinely verified that $\left(T_{n}\right)$ is ergodic (resp. weakly mixing) iff for every $A \in \mathscr{B}$ with $\mu(A)<\infty$ the sequence $\left(T_{n} 1_{A}\right)$ is ergodic (resp. weakly mixing) in $L^{2}(X, \mathscr{B}, \mu)$. Now for a sequence of the form $\left(T_{n} 1_{A}\right)$ both ergodicity and weak mixing are equivalent to the condition

$$
\frac{1}{N^{2}} \sum_{m, n=1}^{N} \mu\left(T_{m}^{-1} A \cap T_{n}^{-1} A\right) \underset{N \rightarrow \infty}{\rightarrow} 0 .
$$

It follows that $\left(T_{n}\right)$ is weakly mixing iff it is ergodic. We now state

THEOREM 3.2. Let $\left(T_{n}\right)$ be a sequence of measure preserving transformations of a $\sigma$-finite infinite measure space $(X, \mathscr{B}, \mu)$, and $1<p<\infty$. Then $\left(T_{n}\right)$ is weakly mixing iff for every $f \in L^{p}(X, \mathscr{B}, \mu)$ and $\delta, \varepsilon>0$ there exists an $L$ such that, if $N>L$ and $n_{1}<n_{2}<\cdots<n_{N}<N / \delta$, then

$$
\left\|\frac{1}{N} \sum_{k=1}^{N} T_{n_{k}} f\right\|<\varepsilon .
$$

The proof follows that of Theorem 2.1, where we employ Theorem 3.1 instead of Theorem 1.1.

\section{REFERENCES}

1. M. A. Akcoglu and L. Sucheston, On operator convergence in Hilbert space and in Lebesgue space, Period. Math. Hungar. 2 (1972), 235-244.

2. Weak convergence of positive contractions implies strong convergence of averages, $\mathrm{Z}$. Wahrsch. Verw. Gebiete 32 (1975), 139-145.

3. A. Bellow, An $L^{p}$-inequality with applications to ergodic theory, Houston J. Math. 1 (1975), 153-159.

4. D. Berend and V. Bergelson, Ergodic and mixing sequences of transformations, Ergodic Theory Dynamical Systems 4 (1984), 353-366.

5. J. R. Blum and D. L. Hanson, On the mean ergodic theorem for subsequences, Bull. Amer. Math. Soc. 66 (1960), 308-311.

6. H. Fong and L. Sucheston, On a mixing property of operators in $L_{p}$-spaces, Z. Wahrsch. Verw. Gebiete 28 (1974), 165-171.

7. L. K. Jones, A mean ergodic theorem for weakly mixing operators, Adv. in Math. 7 (1971), 211-216. 
8. L. K. Jones and V. Kuftinec, A note on the Blum-Hanson theorem, Proc. Amer. Math. Soc. 30 (1971), 202-203.

9. L. K. Jones and M. Lin, Ergodic theorems of weak mixing type, Proc. Amer. Math. Soc. 57 (1976), 50-52.

10. V. Krengel and L. Sucheston, On mixing in infinite measure spaces, $\mathrm{Z}$. Wahrsch. Verw. Gebiete 13 (1969), 150-164.

11. D. A. Raikov On some arithmetical properties of summable functions, Mat. Sb. 1 (43) (1936), 377-384. (Russian)

Department of Mathematics, University of California, los Angeles, California 90024

Department of Mathematics, Ohio State Univerersity, Columbus, Ohio 43210 (Current address of Vitaly Bergelson)

Current address (Daniel Berend): Department of Mathematics and Computer Science, Ben-Gurion University, Beer Sheva 84105, Israel 